Presented at the:

"Fifth Williamsburg Workshop on First-Principles Calculations for Ferroelectrics", February 1-4, Williamsburg, VA, USA

\title{
Hartree-Fock Studies of the Ferroelectric Perovskites
}

\author{
L. Fu, ${ }^{1}$ E. Yaschenko, ${ }^{1}$ L. Resca, ${ }^{1}$ and R. Resta ${ }^{1,2}$ \\ ${ }^{1}$ Department of Physics, The Catholic University of America, Washington, D.C. 20064 \\ ${ }^{2}$ INFM-Dipartimento di Fisica Teorica, Università di Trieste, Strada Costiera 11, I-34014 \\ Trieste, Italy
}

\begin{abstract}
Within an ab-initio HF scheme, we use both Berry-phase calculations and supercell calculations in order to compute the dynamical charges for lattice dynamics and the electronic dielectric constant for $\mathrm{KNbO}_{3}$ and $\mathrm{BaTiO}_{3}$. Comparison with experimental data indicates that HF provides a description of the electronic properties of this material whose accuracy is of the same order as the LDA one. There are however significant differences between the two sets of results, whose origin is scrutinized. Motivated by the study of surface and domain-boundary properties, we also present some results for $\mathrm{BaTiO}_{3}$ slabs, including both genuinely isolated and periodically repeated slabs with different terminations. The capability of dealing with a genuinely isolated slab is a virtue of the localized-basis implementation adopted here. We demonstrate, amongst other things, the nontrivial dynamical-charge neutrality of $\mathrm{BaTiO}_{3}[001]$ surfaces.
\end{abstract}

\section{INTRODUCTION}

Density-functional theory (DFT) has been very successful in predicting several physical properties of the ferroelectric perovskites, most implementations being performed within the local-density approximation (LDA). Here we assess the performance of the alternative Hartree-Fock (HF) scheme for these materials, which have an intermediate ionic/covalent character. In general, DFT is particularly convenient and accurate for metals and semiconductors [1], while the HF scheme is very reliable for ionic crystals, and insulators in general $[2,3]$. In this paper, we address at the HF level some fundamental dielectric properties, such as the dynamical ionic charges and the dielectric constant, in two paradigmatic ferroelectric perovskites: $\mathrm{KNbO}_{3}$ and $\mathrm{BaTiO}_{3}$.

The very first HF study of a ferroelectric perovskite, at the ab-initio level, has been published very recently [4]. The case study was $\mathrm{KNbO}_{3}$, and several properties of the electronic ground state were investigated, including the broken-symmetry instability of the tetragonal structure of this material. In this same work the Berry-phase theory of macroscopic polarization $[5,6]$ is implemented within the 
HF scheme, using first-principles ingredients. Perhaps the most interesting result that has emerged is that the calculated $\mathrm{HF}$ spontaneous polarization of $\mathrm{KNbO}_{3}$ in its tetragonal phase is $0.34 \mathrm{C} / \mathrm{m}^{2}$, quite close to the experimental value [7], which is $0.37 \mathrm{C} / \mathrm{m}^{2}$, as well as previous LDA results [8-10], which are between 0.33 and $0.40 \mathrm{C} / \mathrm{m}^{2}$.

Although the computed value of the spontaneous polarization is almost identical within LDA and HF, we will show that there are significant differences when the dynamical charges of the ions are separately investigated. These polarizabilities are dominated by covalency effects, which plausibly are described in different ways at the HF and LDA levels.

We will then show that, by combining results from both Berry-phase calculations and supercell calculations, one has access to the theoretical value of the electronic dielectric constant $\varepsilon_{\infty}$ [11]. It is well known that LDA overestimates the value of $\varepsilon_{\infty}$ in any material, whereas it has been only guessed that HF underestimates it, since no calculation was previously available, for any material. Our HF calculated $\varepsilon_{\infty}$ of $\mathrm{KNbO}_{3}, \mathrm{BaTiO}_{3}$ and $\mathrm{MgO}$ are indeed smaller than experimental values.

Finally, we will show some results for $\mathrm{BaTiO}_{3}$ slabs, including both isolated and periodic slabs with different terminations. Since most first-principles schemes are implemented within plane-wave based computational methods, they are very effective for infinite periodic systems but cannot treat the isolated slabs. Even the periodic slabs with moderate vacuum separations prove to be a heavy computational burden for plane-wave methods, and only a few such calculations have been performed for ferroelectric perovskites $[12,13]$. The capability of dealing with a genuinely isolated slab is a virtue of the localized-basis implementation adopted here $[2,14]$. We will discuss bulk dielectric properties, surface charge, surface energies, work function, and surface dynamical charges. We will show how the computed values of these quantities change as functions of the number of atomic layers and the thickness of the vacuum layers separating the periodic slabs. Owing

to a recently discovered theorem, the dynamical charge of a given ion at a polar surface cannot have the same value as in the bulk [15].

\section{BULK DYNAMICAL CHARGES AND DIELECTRIC CONSTANT}

The transverse (Born) charge tensor is defined via the macroscopic polarization $\Delta \mathbf{P}$ linearly induced by a rigid displacement $\mathbf{u}_{s}$ of the $s$ sublattice, while the field is kept at zero value [16]. Namely,

$$
\Delta P_{\alpha}=\frac{1}{\Omega} \sum_{\beta} Z_{\alpha \beta}^{*(\mathrm{~T})} u_{\beta},
$$

where $\Omega$ is the cell volume, and atomic units are used throughout. The longitudinal (Callen) charge tensor $Z^{*(\mathrm{~L})}$ is similarly defined, but with the sublattice 


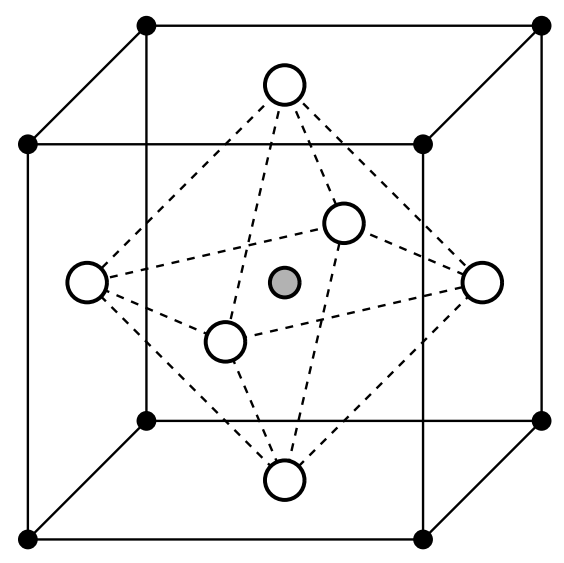

Figure 1. Cubic perovskite structure, with general formula $\mathrm{ABO}_{3}$, where in our cases $\mathrm{A}$ is either $\mathrm{K}$ or $\mathrm{Ba}$ (solid circles) and $\mathrm{B}$ is either $\mathrm{Nb}$ or $\mathrm{Ti}$ (shaded circle). The oxygens (empty circles) form octahedral cages, with $\mathrm{B}$ at their centers, and arranged in a simple cubic pattern. We will consider either displacements in the vertical direction, or slabs with horizontal surfaces. In both cases "O $\mathrm{O}_{\mathrm{I}}$ " refers to top and bottom oxygen ions and "O II" refers to the four oxygen ions in the basal plane of the octahedron. We have therefore $\mathrm{AO}_{\mathrm{I}}$ and $\mathrm{BO}_{\mathrm{II}}$ planes.

displacement performed in a depolarization field $\Delta \mathbf{E}=-4 \pi \Delta \mathbf{P}$. The relation between the two dynamical charges is

$$
Z^{*(\mathrm{~T})}=\epsilon_{\infty} Z^{*(\mathrm{~L})},
$$

where $\epsilon_{\infty}$ is the dielectric constant in the relevant direction. In general, the dynamical charge can be very different from the nominal static charge of an ion. The differences are particularly dramatic when the material has a mixed ioniccovalent character, such as the materials chosen in the present work $[17,18]$.

We calculate the $Z^{*(\mathrm{~T})}$ as a Berry-phase $[5,6]$, while we calculate the $Z^{*(\mathrm{~L})}$ using a supercell technique. The latter approach was introduced by Martin and Kunc several years ago to compute dynamic charges in semiconductors [19], and is still very much in use today in the framework of LDA pseudopotential calculations with plane-wave basis sets. The same supercell technique in the framework of HF calculations with a localized basis set was implemented for the first time in Ref. [11]. Since the aim of the present work is to assess the HF values versus LDA ones, we only present here results for the cubic structure. For $\mathrm{KNbO}_{3}$ we use the lattice constant $4.016 \AA$. This is the same value used in the LDA study of Ref. [10], which is based on the low-temperature experimental data [7]. For $\mathrm{BaTiO}_{3}$, we use the lattice constant $4.006 \AA$. Figure 1 shows the structure of these perovskites.

We use the CRYSTAL95 code, which implements both the HF and LDA self consistent scheme with a linear-combination-of-atomic-orbitals (LCAO) basis set [14]. In our supercell calculations, we displace the sublattice of an atom in the supercell lattice, and compute the planar average of the charge density, potential, and field. We then perform additionally the macroscopic average defined as in Ref. [20], which proves an invaluable tool in the present business. The output of the supercell calculations provide the longitudinal dynamical charges. We use both 3-fold and 4-fold supercells in our supercell calculations, which yield almost identical results. We refer to the original paper for more details [11].

In our Berry-phase calculations, we compute the induced macroscopic polarization $\Delta \mathbf{P}$ in zero field, and thus the corresponding transverse dynamical charges. 


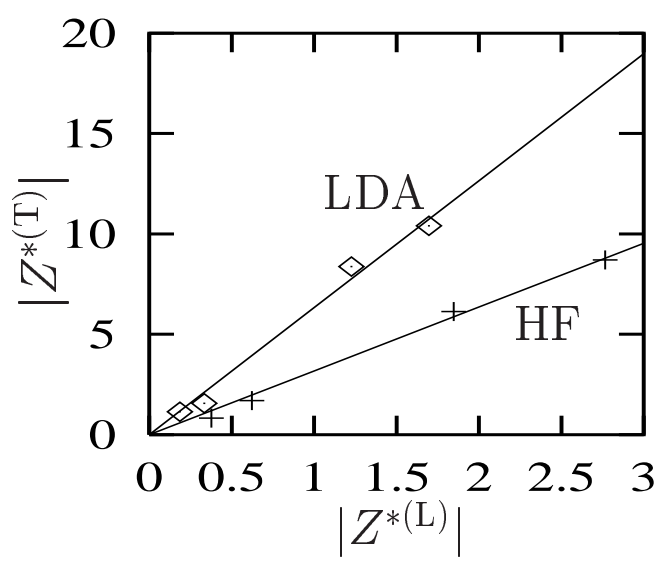

Figure 2. The transverse charge $\left|Z^{*(\mathrm{~T})}\right|$ versus longitudinal charge $\left|Z^{*(\mathrm{~L})}\right|$. The slopes of the two solid lines are, respectively, the LDA and HF dielectric constant $\epsilon_{\infty}$.

In order to compare with the LDA results and examine the performance of our localized basis set, we also run the CRYSTAL95 code in the LDA option for $\mathrm{KNbO}_{3}$. Our results for $\mathrm{KNbO}_{3}$ dynamic charges are reported in Table I.

Table I. Dynamical charges for $\mathrm{KNbO}_{3}$

\begin{tabular}{||l|c|c|c|c|c||}
\hline & $\mathrm{K}$ & $\mathrm{Nb}$ & $\mathrm{O}_{\mathrm{I}}$ & $\mathrm{O}_{\text {II }}$ & sum \\
\hline $\mathrm{Z}^{*(\mathrm{~L})}, \mathrm{HF}, 3$-fold supercell & 0.377 & 2.766 & -1.848 & -0.624 & 0.047 \\
\hline $\mathrm{Z}^{*(\mathrm{~L})}$, LDA, 3-fold supercell & 0.187 & 1.696 & -1.227 & -0.335 & -0.014 \\
\hline $\mathrm{Z}^{*(\mathrm{~T})}, \mathrm{HF}$, Berry-phase & 0.830 & 8.702 & -6.130 & -1.703 & -0.004 \\
\hline $\mathrm{Z}^{*(\mathrm{~T})}$, LDA, Berry-phase & 1.144 & 10.400 & -8.377 & -1.567 & 0.033 \\
\hline $\mathrm{Z}^{*(\mathrm{~T})}$, LDA, Ref. [10] & 1.12 & 9.67 & -7.28 & -1.74 & 0.03 \\
\hline
\end{tabular}

The last column shows that the acoustic sum rule [16] is satisfactorily obeyed. A few calculations for $Z^{*(\mathrm{~T})}$ of $\mathrm{KNbO}_{3}$ at $\mathrm{LDA}$ level exist [8-10]: these data are slightly scattered and in some cases refer to slightly different geometries. Here we compare our results with Ref. [10], which uses the same geometry. Our LDA $Z^{*(\mathrm{~T})}$ are in reasonable agreement with those of Ref. [10]. The small differences may be due to the fact that our LDA exchange-correlation functional differs somewhat from that of Ref. [10], and our pseudopotentials and basis sets are optimized for HF rather than LDA.

To determine the dielectric constant $\epsilon_{\infty}$, we plot $\left|Z^{*(\mathrm{~T})}\right|$ versus $\left|Z^{*(\mathrm{~L})}\right|$, and use a least-square fitting to determine the slope which yields $\epsilon_{\infty}$. This is shown in Fig. 2 , which indicates that the correlation between our $Z^{*(\mathrm{~L})}$ and $Z^{*(\mathrm{~T})}$ is reasonably linear. For $\mathrm{KNbO}_{3}$ we obtain the LDA value $\epsilon_{\infty}=6.33$, which is close to the LDA value of 6.63 obtained in Ref. [10], and is about $35 \%$ higher than the experimental value 4.69. Similarly, we obtain the $\mathrm{HF}$ value $\epsilon_{\infty}=3.17$, which is about $32 \%$ smaller than the experimental value.

Table II. Dynamical charges for $\mathrm{BaTiO}_{3}$

\begin{tabular}{||l|c|c|c|c|c||}
\hline & $\mathrm{Ba}$ & $\mathrm{Ti}$ & $\mathrm{O}_{\mathrm{I}}$ & $\mathrm{O}_{\text {II }}$ & sum \\
\hline $\mathrm{Z}^{*(\mathrm{~L})}, \mathrm{HF}, 3$-fold supercell & 0.909 & 2.126 & -1.468 & -0.797 & -0.027 \\
\hline $\mathrm{Z}^{*(\mathrm{~T})}, \mathrm{HF}$, Berry-phase & 2.052 & 5.996 & -4.319 & -1.846 & -0.037 \\
\hline $\mathrm{Z}^{*(\mathrm{~T})}$, LDA, Ref. [21] & 2.74 & 7.29 & -5.75 & -2.13 & 0.02 \\
\hline
\end{tabular}


We have performed analogous calculations for $\mathrm{BaTiO}_{3}$, whose results are reported in Table II: the resulting HF dielectric constant is $\epsilon_{\infty}=2.76$. This value is about one half of the experimental value 5.40. The LDA value reported in Ref. [21], for a slightly different lattice constant, is 6.73 . We have also performed a series of similar calculations for the more simple oxide $\mathrm{MgO}$ [22]. We use the cubic lattice constant $a=4.21 \AA$. We obtain HF $Z^{*(\mathrm{~L})}= \pm 1.267$ and $Z^{*(\mathrm{~T})}= \pm 1.809$, which yields a $\mathrm{HF}$ $\epsilon_{\infty}=1.428$. This value is about one half of the experimental value 2.94 , while the LDA value reported in Ref. [23] is 3.14 .

As a general conclusion about the study of bulk dielectric properties, we find that the calculated transverse dynamical charges are about of the same quality within $\mathrm{HF}$ and LDA, the trend being that the HF ones are generally closer to the nominal value of the corresponding static charges. This seems to indicate that HF tends to underestimate the covalence mechanism, while LDA tends to overestimate it. The resuts for the dielectric constants confirm this same message.

\section{SURFACE PROPERTIES VIA SLAB CALCULATIONS}

We now switch to the HF investigation of several surface properties of $\mathrm{BaTiO}_{3}$. To this aim we have used both isolated slabs and periodically repeated ones, while at this preliminary stage surface geometries have not been relaxed: all results refer therefore to ideal (truncated bulk) geometries. There are two major differences between the two kinds of calculations. First of all, there are interactions among the periodic slabs, which presumably vanish when the vacuum separating the slabs is sufficiently thick. Using a basis of plane waves (or plane-wave-like, such as LAPW), the increase in the vacuum space drastically increase the number of plane waves necessary for convergence, and therefore the computational workload. Secondly, the isolated slabs are calculated by imposing the boundary condition of vanishing external field, while the periodic slabs are necessarily calculated with the boundary condition of vanishing average field (over the supercell). This is a major problem when dealing with supercells built of asymmetrically terminated slabs, even in absence of macroscopic polarization. The reason is that the work functions of the two surfaces are generally different. The supercell is an artificial periodic structure of repeated slabs and vacuum layers, where the electrostatic potential is enforced

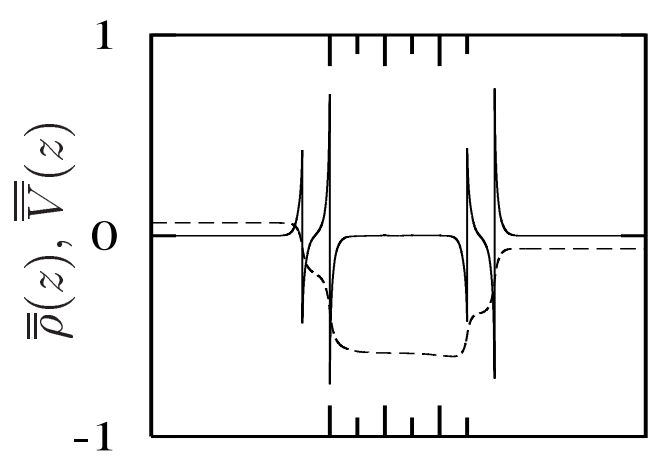

Figure 3. The macroscopic average of charge density $\left(10^{-1}\right.$ electron/bohr ${ }^{3}$, solid line) and potential (hartree, dashed line) for an isolated slab of six atomic layers, where the bulk structure is centrosymmetric. The vertical bars indicate the positions of the atomic layers: short bars for $\mathrm{BaO}_{\mathrm{I}}$ planes, long bars for $\mathrm{TiO}_{\mathrm{II}}$ planes. 


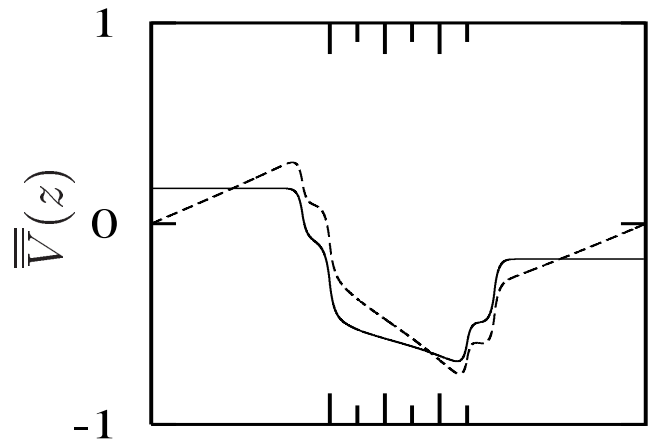

Figure 4. Macroscopic averages of the potential (hartree) for an isolated slab (solid line) and a periodically repeated slab (dashed line). Both calculations refer to slabs cut from a noncentrosymmetric bulk having the experimental ferroelectric distortions. The meaning of vertical bars is the same as in Fig. 3 .

to be periodical. This periodicity, combined with the vacuum-level difference (due to the different work functions), necessarily originates fictitious electric fields and static surface charges. Such unphysical charges can in principle be made arbitrarily small by increasing the thickness of the vacuum layers, but in practice a large enough supercell may be computationally unaffordable.

The problem does not occur when performing calculations for isolated slabs within a localized basis set. As an example we plot in Fig. 3 the macroscopic average of charge density, potential, and field for an isolated slab with asymmetric termination, where however the slab has been cut from a centrosymmetric bulk. There are several very perspicuous features to remark. (1) First of all, despite the asymmetric termination of the slab of Fig. 3, the macroscopic field vanishes both in the bulk region and in the vacuum region. This means that each of the two surfaces is neutral, as required by very general considerations. (2) One observes some structure only in the surface regions. Basically, there is a quadruple charge layer (two double layers) at each surface. We also see that in the middle region of the slab both the charge and field vanish and the potential remains constant. This means that the bulk like behavior is recovered in the middle region of the slab. (3) Although the field in the vacuum regions is zero, the potential goes to two different limits to the left and to the right of the sample. This net change in the potential across the slab is precisely the difference in the work functions of the two nonequivalent surfaces, discussed above. (4) We have additionally calculated all kinds of slabs (asymmetrically terminated as in Fig. 3, doubly Ti-terminated, doubly Ba-terminated): all calculated surface properties look precisely the same, while instead they artificially do depend on the choice of the slab in the periodically repeated case.

As a matter of fact, periodically repeated slabs are systematically messed up by the above problems. We show here an example in Fig. 4, where the occurrence of the fictitious field is very perspicuous, despite the large thickness of the vacuum region (6 lattice constants in this calculation). Because of this major problem, very few calculations of a periodically repeated slab (asymmetrically terminated) have ever been performed, for any material. For a discussion of the difficulties, see Refs. $[12,15]$. By contrast with this, the isolated-slab calculation conveys a very clear message, and the slope of the potential in the bulk region of Fig. 4 is no artifact: the presence of a macroscopic field is a physical feature, related to 


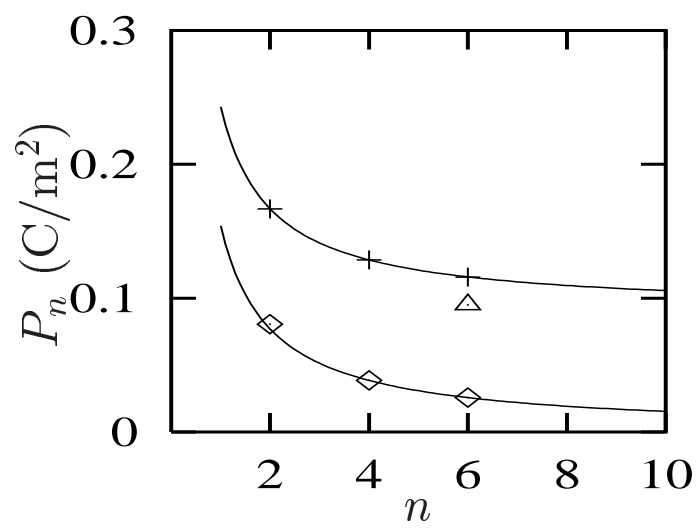

Figure 5. Dipole moment per unit volume as functions of the number of atomic layers, for isolated centrosymmetric (diamonds) and ferroelectric (crosses) slabs. The solid lines are fits of Eq. (4). The triangle is the bulk polarization calculated from the field in the bulk region of the ferroelectric slab.

the spontaneous polarization of the bulk material. This will be discussed in the following.

\section{Polarization, surface charge, and surface dipoles}

Let us consider the total dipole per unit area of a finite slab of $n$ atomic layers: this dipole is easily evaluated from isolated-slab calculations simply looking at the net change in potential across the slab $\Delta V_{\text {slab }}$, and is obviously nonzero even for centrosymmetric slabs (with asymmetric termination), as in Fig. 3. In the case of a ferroelectric slab, an additional contribution to the dipole comes from the spontaneous polarization. In the limit of large $n$, one must recover the appropriate bulk polarization. Since the nominal thickness of the slab is $n a / 2$, we write the dipole per unit volume as:

$$
P_{n}=\frac{\Delta V_{\text {slab }}}{2 \pi n a} \simeq \sigma_{0}+\frac{2 p_{0}}{n} .
$$

In the latter expression $\sigma_{0}$ is the charge per unit area which accumulates at the slab surface, and is a bulk property dependent on the spontaneous polarization of the material. Instead $p_{0}$ is proportional to the work function difference discussed above and is therefore a surface property. Our results are displayed in Fig. 5, which shows the asymptotic behavior of $P_{n}$ : the surface charge $\sigma_{0}$ is clearly zero in the centrosymmetric case, while we estimate $P_{\infty}=\sigma_{0}=0.089 \mathrm{C} / \mathrm{m}^{2}$ for the ferroelectric isolated slab.

Besides the above asymptotic extrapolation, an alternative path to evaluating $\sigma_{0}$ consists in measuring the macroscoic electric field in the middle of the isolated slab. In the centrosymmetric case we get zero field at any thickness, while the value of $\sigma_{0}$ extracted in this way from the ferroelectric slab calculation, shown in Fig. 5 as a triangle, is $\sigma_{0}=0.095 \mathrm{C} / \mathrm{m}^{2}$, in good agreement with the above mentioned value.

Finally, we wish to compare the above calculated value of $P_{\infty}$ to the bulk spontaneous polarization of the material, as measured in experiments and as previously calculated within LDA $[9,21]$. It is important to notice that our $P_{\infty}$ is by definition the macroscopic polarization in a depolarizing field, while the literature 
generally refers to the spontaneous polarization in zero field. The latter is related to the former by a factor $\varepsilon_{\infty}$, much in the same way as the longitudinal and transverse dynamical charges in Eq. (2).

Using our theoretical value of 2.76 for the $\mathrm{HF}$ dielectric constant we get a spontaneous polarization of $0.245 \mathrm{C} / \mathrm{m}^{2}$, which compares extremely well to the experimental value of $0.263 \mathrm{C} / \mathrm{m}^{2}$ [24], and to LDA values in the range 0.286 to $0.363 \mathrm{C} / \mathrm{m}^{2}[9,21]$.

In order to achieve a more meaningful comparison, we have also run our own Berry phase calculation using identical technical ingredients and geometry as in the slab calculations. We get a value of $0.240 \mathrm{C} / \mathrm{m}^{2}$, in very good agreement with the above evaluation of the same quantity from the electric field inside the isolated slab. This shows the internal consistency of the HF properties, calculated at the HF level. Indeed, the small disagreement provides an estimate of the numerical error, within the same given physical approximation. We emphasize that, on the contrary, any periodic slab calculation would be affected by much larger numerical errors.

\section{Surface energy}

We report here a study of the surface energies. We emphasize that - since the surface geometries have not been relaxed - our preliminary calculated values have not to be taken as realistic. What we demonstrate instead is that using a genuinely isolated slab we have a fast convergence to the genuine surface properties, whereas convergence is rather slow in the periodic-slab geometry. Our slabs, made of $n$ atomic layers cut from a centrosymmetric bulk, have symmetric termination for odd $n$, and asymmetric termination for even $n$.

In the present [001] geometry two nonequivalent truncations of the bulk crystal are obviously possible. Whenever this happens - as thoroughly discussed in the literature [25] - the surface energy of each of the nonequivalent surfaces is ill defined and any stability issue must be studied by introducing the appropriate chemical potential. We therefore address the average surface energy (over the two

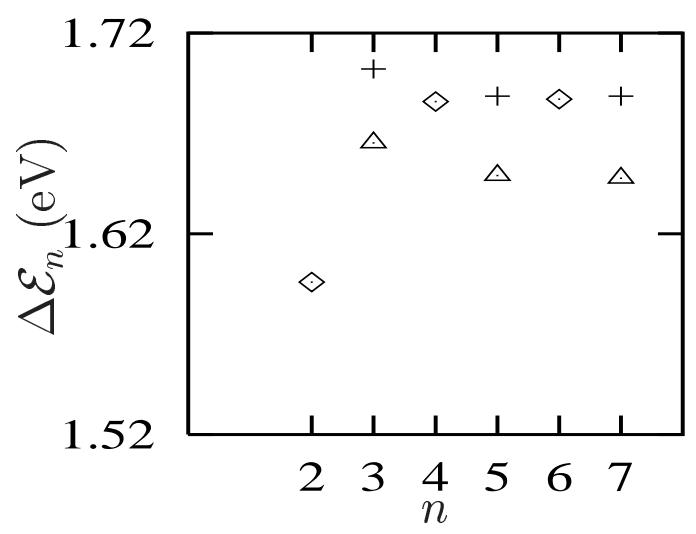

Figure 6. Calculated surface energy as functions of the number of atomic layers. The diamonds and crosses are, respectively, the results from isolated slabs with asymmetric and symmetric terminations. The triangles are the results from periodic slabs with symmetric terminations. 
nonequivalent surfaces).

The average surface energy $\Delta \mathcal{E}$ per surface unit cell can be evaluated from slab calculations (with $n$ atomic layers) as the asymptotic value of

$$
\Delta \mathcal{E}_{n}= \begin{cases}{\left[2 \mathcal{E}_{n}-n \mathcal{E}_{\text {bulk }}\right] / 4,} & \text { even } n, \\ {\left[\mathcal{E}_{n}(\mathrm{Ti})+\mathcal{E}_{n}(\mathrm{Ba})-n \mathcal{E}_{\text {bulk }}\right] / 4,} & \text { odd } n,\end{cases}
$$

where $\mathcal{E}_{n}(\mathrm{Ti})$ and $\mathcal{E}_{n}(\mathrm{Ba})$ indicate the energy of a symmetrically terminated slab with $\mathrm{Ti}$ or $\mathrm{Ba}$ termination, respectively.

From Fig. 6 we see that for isolated slabs, as $n$ increases, the average surface energy converges very fast to the asymptotic value: from above for odd $n$, and from below for even $n$. On the other hand, the results for periodic slabs are sistematically lower than those of isolated slabs. This indicates that interactions among slabs still exist, even with a large vacuum separation: in all calculations reported here, we use a fixed supercell size of 12 interlayer spacings. We mention that the surface energies obtained in previous calculations at LDA level using periodic slabs are $0.921 \mathrm{eV}$ [12]) and $1.237 \mathrm{eV}$ [13] for a relaxed geometry [12]), while $1.358 \mathrm{eV}$ is reported for the unrelaxed geometry [13]. As stated above, our geometry is unrelaxed: the data in Fig. 6 extrapolate to $1.69 \mathrm{eV}$.

\section{Surface dynamical charges}

We evaluate the surface longitudinal dynamical charges $\mathrm{Z}^{*(\mathrm{~L})}$ of $\mathrm{BaTiO}_{3}$ from $\mathrm{HF}$ calculations performed on symmetrically-terminated slabs of seven atomic layers. The bulk is centrosymmetric, and the reference surface geometry is not relaxed. Upon displacing atoms in the surface region, we get the results in an analogous way as for the bulk longitudinal charges discussed above. The dynamical charges of ions in the first and second outermost surface layer are reported in Tab. III, together with the corresponding bulk values (taken from Tab. II), for the sake of comparison.

Table III. Surface $Z^{*(\mathrm{~L})}(\mathrm{HF})$

\begin{tabular}{||c|c|c|c|c|c|c||}
\hline & $\mathrm{Ba}$ & $\mathrm{Ti}$ & $\mathrm{O}_{\mathrm{I}}$ & $\mathrm{O}_{\mathrm{II}}$ & $\mathrm{Ba}+\mathrm{O}_{\mathrm{I}}$ & $\mathrm{Ti}+2 \mathrm{O}_{\text {II }}$ \\
\hline first layer & 1.104 & 2.093 & -1.354 & -0.919 & -0.250 & 0.255 \\
\hline second layer & 0.911 & 2.119 & -1.473 & -0.767 & -0.562 & 0.585 \\
\hline bulk & 0.883 & 2.187 & -1.543 & -0.765 & -0.660 & 0.657 \\
\hline
\end{tabular}

We notice that $\mathrm{BaO}_{\mathrm{I}}$ planes and $\mathrm{TiO}_{\mathrm{II}}$ planes are nominally charge neutral, in the hypothesis of complete ionicity. But dynamical charges-particularly in a material having mixed ionic-covalent character as the present one-are dramatically different from the static nominal ones. As a matter of fact, the data in the last two columns of Tab. III show that $\mathrm{BaO}_{\text {I }}$ planes and $\mathrm{TiO}_{\text {II }}$ planes are far from being dynamically neutral, both in the bulk and in the surface region. 
As observed above, the surface of a centrosymmetric slab is charge-neutral as far as the static charge accumulated in the surface region is concerned. This fact implies - owing to a recently discovered theorem [15] - that the surface region must also be dynamically neutral. We notice that the dynamical charges in the surface region deviate considerably from their bulk value, although they converge rather fast to it. A corollary of the dynamical-neutrality theorem, as applied to the [001] geometry considered here, states the the sum of the dynamical charges in the surface planes must add up to one half of the corresponding bulk value (with the appropriate sign) [15]. This sum rule - which can be regarded as the surface analogue of the popular acoustic sum rule for the bulk [16] — is very accurately fullfilled by our calculations. In fact summing over the two outermost surface layers we get $-0.250+0.585=0.335$ and $0.255-0.562=-0.307$ for the two nonequivalent surfaces. Both figures are indeed very close (in modulus) to one half of the dynamical charge of a given bulk plane.

\section{CONCLUSIONS}

We have reported several ab-initio HF results concerning bulk and surface properties of two important ferroelectric perovskites: $\mathrm{KNbO}_{3}$ and $\mathrm{BaTiO}_{3}$. Though

some of our results are preliminary, they indeed assess the accuracy of both the physical approximations and the computational implementation.

About the physical approximations, comparison of our results with the experiment indicates an overall trend: the HF results are about of the same quality as - but on the opposite side of - the LDA results. This trend makes HF an appealing method for "bracketing" a theoretical prediction: it is well exemplified by the case study of the dielectric constant $\varepsilon_{\infty}$ discussed here for $\mathrm{KNbO}_{3}, \mathrm{BaTiO}_{3}$, and even for the simpler oxide $\mathrm{MgO}$.

About the computational implementation within a localized basis set, we have emphasized its merits by showing some case studies of surface properties, where we can study genuinely isolated slabs in vacuo. This is at variance with basically all previous first-principle investigation of surface properties which, for any material, were typically performed within supercell (i.e. periodically repeated slab) geometries.

\section{ACKNOWLEDGMENTS}

We are indebted to S. Dall'Olio and R. Dovesi for discussions and help in using CRYstal95, as well as for providing us with optimized values of the bulk computational parameters. This work is supported by the Office of Naval Research, through grant N00014-96-1-0689. 


\section{REFERENCES}

1. M.L. Cohen, in Electronic Materials, edited by J.R. Chelikowsky and A. Franciosi (Springer, Berlin, 1991), p.57.

2. C. Pisani, R. Dovesi, and C. Roetti, Hartree-Fock Ab-Initio Treatment of Crystalline Systems, Lecture Notes in Chemistry, Vol. 48 (Springer, Berlin, 1988).

3. Quantum-Mechanical Ab-initio Calculation of the Properties of Crystalline Materials, Lecture Notes in Chemistry, Vol 67, edited by C. Pisani (Springer, Berlin, 1996).

4. S. Dall'Olio, R. Dovesi, and R. Resta, Phys. Rev. B 56, 10105 (1997).

5. R. Resta, Ferroelectrics 136, 51 (1992); R.D. King-Smith and D. Vanderbilt, Phys. Rev. B 47, 1651 (1993); R. Resta, Europhys. Lett. 22, 133 (1993).

6. R. Resta, Rev. Mod. Phys. 66, 809 (1994).

7. M.D. Fontana, G. Métrat, J.L. Servoin, and F. Gervais, J. Phys.C 17, 483 (1984); W. Kleemann, F.J. Schäfer, and M.D. Fontana, Phys. Rev. B 30, 1148 (1984).

8. R. Resta, M. Posternak, and A. Baldereschi, Phys. Rev. Lett. 70, 1010 (1993).

9. W. Zhong, R.D. King-Smith, and D. Vanderbilt, Phys. Rev. Lett. 72, 3618 (1994).

10. C.Z. Wang, R. Yu, and H. Krakauer, Phys. Rev. B 54, 11161 (1996).

11. L. Fu, E. Yaschenko, L. Resca, and R. Resta, Phys. Rev. B, 15Mar98.

12. R. E, Cohen, J. Phys. Chem. Solids 57, 1393 (1996); Ferroelectrics 194, 323 (1997).

13. J. Padilla and D. Vanderbilt, Phys. Rev. B 56 , 1625 (1997).

14. R. Dovesi, V.R. Saunders, C. Roetti, M. Causà, N.M. Harrison, R. Orlando, and E. Aprà, CRYstal95 User's Manual, University of Turin (Torino, 1996).

15. A. Ruini, S. Baroni, and R. Resta, Phys. Rev. B, 1Mar98 (preprint condmat/9701194).

16. R. Pick, M.H. Cohen, and R.M. Martin, Phys. Rev. B 1, 910 (1970).

17. M. Posternak, R. Resta, and A. Baldereschi, Phys. Rev. B 50, 8911 (1994).

18. R. Resta, Ferroelectrics 194, 1 (1997).

19. R.M. Martin and K. Kunc, Phys. Rev. B 24, 2081 (1981); K. Kunc, in Electronic Structure, Dynamics and Quantum Structural Properties of Condensed Matter, edited by J.T. Devreese and P. Van Camp (Plenum, New York, 1985), p. 227.

20. A. Baldereschi, S. Baroni, and R. Resta, Phys. Rev. Lett. 61, 734 (1988). A complete account can be found in S. Baroni, R. Resta, A. Baldereschi, and M. Peressi, in: Spectroscopy of semiconductor microstructures, edited by G. Fasol, A. Fasolino and P. Lugli, NATO ASI Series B, vol 206 (Plenum Publishing, New York, 1989), p 251.

21. P. Ghosez, Ph.D thesis, Universite Catholique de Louvain, 1997.

22. E. Yaschenko, L. Fu, L. Resca, and R. Resta, Phys. Rev. B, in press.

23. O. Schütt, P. Pavone, W. Windl, K. Karch, and D. Strauch, Phys. Rev. B. 50, 3746 (1994).

24. H.H. Wieder, Phys. Rev. 99, 1161 (1955).

25. G.-X. Qian, R.M. Martin and D.J. Chadi, Phys. Rev. B 38, 7649 (1988). 